\title{
Proposed code of practice for radio- graphers in the event of suspected non-accidental injury (NAI) in children
}

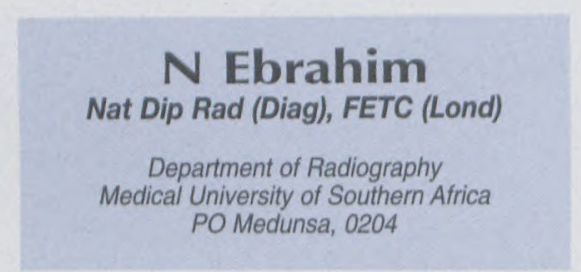

\section{Introduction}

This proposed Code of Practice was developed following a research project conducted in 1999/2000 among radiographers attached to public and private hospitals in South Africa in order to document and record their imaging and management strategies for children with suspected non-accidental injury (NAI).

A 23-item postal questionnaire was sent to 50 randomly selected hospitals. The response rate was $42 \%$. Seventy-five per cent of the respondents saw patients with a history of confirmed NAI, and $70 \%$ saw patients with a history of suspected abuse. Seventy-six per cent of the respondents requested a copy of a Code of Practice for their departments.

\section{Proposed code of practice}

\section{Introduction}

This document has been developed to give guidance to radiographers working in the field of general radiography and specialised imaging procedures.

The document is intended to be a guide and should be read in conjunction with relevant National Legislation, International Law and the following documents:

- The South African Bill of Rights in the Constitution of the Republic of South Africa, Act 108 of 1996.

- Prevention of Family Violence Act 133 of 1993: Section 4.

- The Convention of The Rights of the Child, 2 September 1990 article 49.

- Child Care Act 74 of 1983: Sečtion 42.

\section{Legal considerations}

\section{Consent}

Where invasive imaging procedures are required:

- A parent provides written consent if the child is under the age of 14 years (Child Care Act 74 of 1983: Section 39).

- In the absence of a parent or an official guardian, the medical superintendent may sign the consent form in the case of a medical emergency such as the child with a subdural haematoma (S39).

- If the medical superintendent is not available, the attending medical practitioner acts on behalf of the child as an unauthorised agent. Strict common law requirements, including the law of delict govern the action of such a practitioner.

\section{Confidentiality}

Confidentiality must be maintained as prescribed by the Health Professions Council of South Africa's

\section{Code of Conduct}

In the case of suspected NAI, the radiographer should consult with his/her superior, or the referring practitioner, whichever is appropriate.

Safety

The general safety considerations that apply to adults also apply to children. However, immobilisation devices must be used with care so as not to exacerbate pre-existing injuries.

\section{Evidence}

Before information can be accepted for use in a court of law it must be judged to be admissible evidence. To be admissible, the evidence must be properly authenticated. In order to meet this requirement, the following procedure should be followed:

- Images must be properly identified prior to processing.

- Images must be labelled, preferably by utilising photographic identification cameras. 


\section{TIPS FOR THE RADIOLOGIST}

- In the case where the identification details are not visible, they may be written on the radiograph, accompanied by the radiographer's initials.

- All patient details, together with the correct anatomical marker, must appear on the radiographs.

\section{Continuing evidence}

\section{The radiographic procedure}

- The entire examination must be witnessed - this includes the processing of images. The reports of images must be validated before a Commissioner of Oaths.

- The chain of evidence must be preserved.

- Only primary evidence is admissible in court. In the case of radiographic imaging, it must be original hard copy.

- In the event that copies are needed for medico-legal purposes, such copies must be validated by a Commissioner of Oaths.

- A permanent register should be kept that specifically identifies the radiographer who produced the radiographs plus all other relevant information needed for medicolegal purposes.

- Radiographs should be stored in the X-ray department, and should only be made available to the medical practitioner in charge of the case. A record should be kept of the date and signatures each time radiographs are removed.

\section{Records}

- The original request form, which carries the identity and signature of the radiographer, must be kept in the X-ray department.

- The storage of records must comply with the provisions of the
National Archives of South Africa Act 43 of 1996.

- No radiographic investigation should be undertaken without the original request form.

\section{Digitally stored images}

Courts normally require authenticated original documents to be submitted as evidence. A hard copy for presentation as evidence should be produced at the time of creating a digitally stored image. The digitally stored image can be used as backup evidence if necessary. Electronically produced copies should be validated by a Commissioner of Oaths.

\section{Notification}

In terms of Section 4 of the Prevention of Family Violence Act 133 of 1993 , radiographers are obliged to report child abuse or suspected child abuse to a police officer or a social worker.

The Health Professions Council of South Africa will not institute disciplinary steps against a radiographer who breaches confidentiality in order to comply with the requirement of this Act.

\section{Professional indemnity}

It is recommended that all practising radiographers, including those employed by the state, should take out personal professional indemnity insurance.

\section{Human resources}

\section{Post-traumatic stress syndrome}

Employers need to devise policies for dealing with post-traumatic stress syndrome. Access to a clinical psychologist, psychiatrist, or social worker as the case may be should be provided for staff.

\section{Professional considerations}

- Radiographers should take standard projections of the region(s) of interest and skeletal surveys if they believe them to be necessary.

- The radiographer must keep the radiation dose as low as reasonably practical and must ensure that the child and accompanying adult are not exposed to unnecessary radiation.

- The radiographer must, wherever possible, recommend alternate non-ionising imaging modalities.

- The radiographer must maintain confidentiality.

\section{Radiographic education}

Radiographers should have access to:

- Information on typical NAI injuries.

- Medico-legal information relating to the admissibility of evidence in a court of law and the law of delict in general.

- Information about ethical issues relating to forensic radiography.

\section{Technical aspects}

The role of radiography in NAI is:

- To indicate the locality, nature and extent of injury.

- To confirm or provide evidence with regard to the identity of the patient in the case where a child may have been abandoned.

- To provide evidence of pre-existing skeletal and soft tissue trauma.

- To assist in determining the mechanism of injury.

- To assist in locating ingested, or impaled foreign bodies.

\section{Imaging}

The complexity and severity of the patient's condition should dictate the 


\section{TIPS FOR THE RADIOLOGIST}

selection of the appropriate imaging procedure. The availability of equipment may influence the selection of appropriate imaging modalities.

\section{Protocols}

The protocols in Annexure A have been adapted from those recommended by the American College of Radiology.

\section{Quality assurance}

The skeletal survey should be performed using close collimation of the $\mathrm{X}$-ray beam over the region of interest to improve image contrast. Both joints should be included in all long bone images. Both sides should be imaged for comparison.

\section{Radiographic technical factors}

The KVp should be appropriate for the film screen combination used. The mAs must be adjusted according to the KVp, imaging system, and type of $\mathrm{X}$-ray generator. The shortest possible times should be considered to reduce motional unsharpness.

\section{Digital radiography}

It is important to ensure that the application of digital imaging is comparable with high detail film screens. The system must be capable of detect- ing subtle fractures that are highly specific for the diagnosis of NAI.

\section{Patient restraint and positioning}

As in the case of radiography, proper restraints are essential for high quality images. Wide hypo-allergic radiolucent tape is recommended in the absence of wide binding tape immobilisation that is often supplied with $\mathrm{X}$-ray equipment.

\section{Lead rubber protective devices}

As in the case of general radiography, lead rubber protective devices should be used with care. The gonads should not be covered when imaging the pelvis for the first time. Lead rubber should be well secured during skeletal survey imaging so that it does not obscure regions of interest.

\section{Acknowledgments}

This document was completed for the partial fulfilment of the requirements for the MSc Radiography programme offered by the Anglia Polytechnic University, Cambridge. I wish to thank them for their comments as well as the following individuals: Professor P W W Coetzer, Head, Department of Community Health, The Medical University of Southern Africa for his assistance with review- ing the document and his assistance with the ethical and legal issues surrounding child abuse; $\mathrm{Mr} \mathrm{T} \mathrm{S} \mathrm{M}$ Moalusi, Head of Department of Radiography, The Medical University of Southern Africa for his constant support; Dr R Fisher, Consultant Radiologist, Red Cross Memorial Children's Hospital, Cape Town for provision of additional material required for the study of NAI in children; Professor H S Schoeman, retired Head of the Department of Mathematics and Statistics, The Medical University of Southern Africa for his assistance with the statistical analysis of the research; the lecturers attached to the Department of Radiography, Port Elizabeth Technikon for their input and suggestions; and Professor L D R Tsatsi, Department of Radiography, Ga-Rankuwa Hospital for his comments on the imaging protocols.

Further enquiries and/or comments may be addressed to: Mrs $\mathrm{N}$ Ebrahim, Box 646, The Medical University of Southern Africa, P O Medunsa, 0204.

References and CD (case studies of NAI in children) may be obtained from the author E-mail:nebrahim@medunsa.ac.za; Tel: (012) 521 4264; Postal address: Department of Radiology, PO Box 646, Medunsa, 0204.

\section{ANEXURE A}

\section{WINDOW 1}

The skeletal survey

$$
\begin{aligned}
& \text { Antero-posterior skull } \\
& \text { Lateral skull } \\
& \text { Lateral cervical spine } \\
& \text { Lateral lumbar spine } \\
& \text { Antero-posterior thorax } \\
& \text { Lateral thorax } \\
& \text { Antero-posterior pelvis }
\end{aligned}
$$

Antero-posterior humeri Antero-posterior forearms Antero-posterior hands Oblique hands Antero-posterior femora Antero-posterior tibiae Dorsi-plantar feet

\section{WINDOW 2}

Suspected Physical Abuse - child under 3 years of age VARIANT 1: No focal signs or symptoms
1. Radio-isotope bone scan
2. Skeletal survey
3. Ultrasound - abdomen
4. MRI brain
5. CT brain 
- In the case where the identification details are not visible, they may be written on the radiograph, accompanied by the radiographer's initials.

- All patient details, together with the correct anatomical marker, must appear on the radiographs.

\section{Continuing evidence}

The radiographic procedure

- The entire examination must be witnessed - this includes the processing of images. The reports of images must be validated before a Commissioner of Oaths.

- The chain of evidence must be preserved.

- Only primary evidence is admissible in court. In the case of radiographic imaging, it must be original hard copy.

- In the event that copies are needed for medico-legal purposes, such copies must be validated by a Commissioner of Oaths.

- A permanent register should be kept that specifically identifies the radiographer who produced the radiographs plus all other relevant information needed for medicolegal purposes.

- Radiographs should be stored in the X-ray department, and should only be made available to the medical practitioner in charge of the case. A record should be kept of the date and signatures each time radiographs are removed.

\section{Records}

- The original request form, which carries the identity and signature of the radiographer, must be kept in the X-ray department.

- The storage of records must comply with the provisions of the
National Archives of South Africa Act 43 of 1996.

- No radiographic investigation should be undertaken without the original request form.

\section{Digitally stored images}

Courts normally require authenticated original documents to be submitted as evidence. A hard copy for presentation as evidence should be produced at the time of creating a digitally stored image. The digitally stored image can be used as backup evidence if necessary. Electronically produced copies should be validated by a Commissioner of Oaths.

\section{Notification}

In terms of Section 4 of the Prevention of Family Violence Act 133 of 1993 , radiographers are obliged to report child abuse or suspected child abuse to a police officer or a social worker.

The Health Professions Council of South Africa will not institute disciplinary steps against a radiographer who breaches confidentiality in order to comply with the requirement of this Act.

\section{Professional indemnity}

It is recommended that all practising radiographers, including those employed by the state, should take out personal professional indemnity insurance.

\section{Human resources}

\section{Post-traumatic stress syndrome}

Employers need to devise policies for dealing with post-traumatic stress syndrome. Access to a clinical psychologist, psychiatrist, or social worker as the case may be should be provided for staff.

\section{Professional considerations}

- Radiographers should take standard projections of the region(s) of interest and skeletal surveys if they believe them to be necessary.

- The radiographer must keep the radiation dose as low as reasonably practical and must ensure that the child and accompanying adult are not exposed to unnecessary radiation.

- The radiographer must, wherever possible, recommend alternate non-ionising imaging modalities.

- The radiographer must maintain confidentiality.

\section{Radiographic education}

Radiographers should have access to:

- Information on typical NAI injuries.

- Medico-legal information relating to the admissibility of evidence in a court of law and the law of delict in general.

- Information about ethical issues relating to forensic radiography.

\section{Technical aspects}

The role of radiography in NAI is:

- To indicate the locality, nature and extent of injury.

- To confirm or provide evidence with regard to the identity of the patient in the case where a child may have been abandoned.

- To provide evidence of pre-existing skeletal and soft tissue trauma.

- To assist in determining the mechanism of injury.

- To assist in locating ingested, or impaled foreign bodies.

\section{Imaging}

The complexity and severity of the patient's condition should dictate the 
selection of the appropriate imaging procedure. The availability of equipment may influence the selection of appropriate imaging modalities.

\section{Protocols}

The protocols in Annexure A have been adapted from those recommended by the American College of Radiology.

\section{Quality assurance}

The skeletal survey should be performed using close collimation of the $\mathrm{X}$-ray beam over the region of interest to improve image contrast. Both joints should be included in all long bone images. Both sides should be imaged for comparison.

\section{Radiographic technical factors}

The KVp should be appropriate for the film screen combination used. The mAs must be adjusted according to the KVp, imaging system, and type of X-ray generator. The shortest possible times should be considered to reduce motional unsharpness.

\section{Digital radiography}

It is important to ensure that the application of digital imaging is comparable with high detail film screens. The system must be capable of detect- ing subtle fractures that are highly specific for the diagnosis of NAI.

\section{Patient restraint and positioning}

As in the case of radiography, proper restraints are essential for high quality images. Wide hypo-allergic radiolucent tape is recommended in the absence of wide binding tape immobilisation that is often supplied with X-ray equipment.

\section{Lead rubber protective devices}

As in the case of general radiography, lead rubber protective devices should be used with care. The gonads should not be covered when imaging the pelvis for the first time. Lead rubber should be well secured during skeletal survey imaging so that it does not obscure regions of interest.

\section{Acknowledgments}

This document was completed for the partial fulfilment of the requirements for the MSc Radiography programme offered by the Anglia Polytechnic University, Cambridge. I wish to thank them for their comments as well as the following individuals: Professor P W W Coetzer, Head, Department of Community Health, The Medical University of Southern Africa for his assistance with review- ing the document and his assistance with the ethical and legal issues surrounding child abuse; $\mathrm{Mr} \mathrm{T} \mathrm{S} \mathrm{M}$ Moalusi, Head of Department of Radiography, The Medical University of Southern Africa for his constant support; Dr R Fisher, Consultant Radiologist, Red Cross Memorial Children's Hospital, Cape Town for provision of additional material required for the study of NAI in children; Professor H S Schoeman, retired Head of the Department of Mathematics and Statistics, The Medical University of Southern Africa for his assistance with the statistical analysis of the research; the lecturers attached to the Department of Radiography, Port Elizabeth Technikon for their input and suggestions; and Professor L D R Tsatsi, Department of Radiography, Ga-Rankuwa Hospital for his comments on the imaging protocols.

Further enquiries and/or comments may be addressed to: Mrs N Ebrahim, Box 646, The Medical University of Southern Africa, P O Medunsa, 0204.

\footnotetext{
References and CD (case studies of NAI in children) may be obtained from the author E-mail:nebrahim@medunsa.ac.za; Tel: (012) 521 4264; Postal address: Department of Radiology, PO Box 646, Medunsa, 0204.
}

\section{ANEXURE A}

\section{WINDOW 1}

The skeletal survey

\begin{tabular}{l|l|}
$\begin{array}{l}\text { Antero-posterior skull } \\
\text { Lateral skull }\end{array}$ & $\begin{array}{l}\text { Antero-posterior humeri } \\
\text { Lateral cervical spine }\end{array}$ \\
Antero-posterior forearms \\
Antero-posterior hands \\
Antero-posterior thorax & Oblique hands \\
Lateral thorax & Antero-posterior femora \\
Antero-posterior pelvis & Antero-posterior tibiae \\
& Dorsi-plantar feet \\
\hline
\end{tabular}

\section{WINDOW 2}

Suspected Physical Abuse - child under 3 years of age VARIANT 1: No focal signs or symptoms

1. Radio-isotope bone scan

2. Skeletal survey

3. Ultrasound - abdomen

4. MRI brain

5. CT brain 


\section{TIPS FOR THE RADIOLOGIST}

\section{WINDOW 3}

Suspected Physical Abuse - child under 3 years of age VARIANT 2: Head trauma by history, no focal findings, no neurological abnormalities

1. Plain skeletal survey

2. CT brain or

3. MRI brain

4. Utrasound abdomen

5. Radio-isotope bone scan if clinical suspicion of abuse is high

6. Full skull series if skeletal survey and CT do not show fractures

\section{WINDOW 4}

Suspected Physical Abuse - child under 3 years of age VARIANT3 - Neurological signs and symptoms, with or without physical abuse

1. Plain X-ray skeletal survey

2. Ultrasound cranium

3. CT brain without or with contrast

4. Plain X-ray skull if skeletal survey and CT scan do not show fractures

5. Radio-isotope imaging of the skeleton if clinical suspicion is high

\section{WINDOW 5}

Child of any age with visceral injuries and/or discrepancies with history

VARIANT 4: Physical and laboratory examination inconclusive

1. Skeletal survey

2. Ultrasound abdomen and pelvis

3. Ultrasound cranium

4. CT scan abdomen and pelvis or MRI abdomen and pelvis

5. MRI abdomen and pelvis

6. CT cranium with or without contrast or MRI brain 